\title{
ON THE TIMOSHENKO THEORY OF TRANSVERSE BEAM VIBRATIONS*
}

\author{
BY \\ C. L. DOLPH \\ University of Michigan
}

1. Introduction. The classical one-dimensional theory of flexural motions of elastic bars is based upon the Bernoulli-Euler equation

$$
E I \frac{\partial^{4} y^{*}}{\partial x^{4}}+\rho \frac{\partial^{2} y^{*}}{\partial t^{2}}=0,
$$

where $y^{*}(x, t)$ is the displacement at time $t$ of a point on the central axis. Equation (1) has long been known to be inadequate for the higher modes of vibration. Although Rayleigh [1] introduced the effect of rotary inertia, the one-dimensional theory did not adequately explain the higher modes until Timoshenko [2], [3], extended it to include the effects of transverse shear deformation. The Timoshenko theory is based upon the following equations:

$$
\begin{gathered}
\rho \frac{\partial^{2} y^{*}}{\partial t^{2}}+\frac{\partial V^{*}}{\partial x}=0 \\
-\frac{\partial M^{*}}{\partial x}+I_{0} \frac{\partial^{2} \beta^{*}}{\partial t^{2}}+V^{*}=0 \\
\frac{\partial \beta^{*}}{\partial x}=\frac{M^{*}}{E I} \\
V^{*}=-K A G \alpha^{*} \\
\frac{\partial y^{*}}{\partial x}=\alpha^{*}+\beta^{*}
\end{gathered}
$$

In these equations $y^{*}(x, t)$ represents the transverse deflection, $M^{*}(x, t)$ the bending moment, $V^{*}(x, t)$ the transverse shear force, $\beta^{*}(x, t)$ the neutral axis slope due to bending, and $\alpha^{*}(x, t)$ the neutral axis slope due to shear. Also, EI represents the flexural rigidity, $K A G$ the shear rigidity, and $I_{0}$ the so-called rotary moment of inertia. $I_{0}$ is equal to $\rho I / A$ where $\rho$ is the mass per unit length, $I$ the area moment of inertia, and $A$ the crosssection area. The first two of the above equations are stress equations of motion, the second two are stress-strain relations, and the last is a geometric strain-displacement relation.

While these equations may be derived in a plausible fashion from the laws of dynamics and of elementary strength of materials, their ultimate justification, as well as the range of their validity, must be deduced from the general equations of elasticity. This has recently been accomplished by Mindlin and Hermann not only for beams but for many other approximate equations of elasticity. While these results have not yet been published, they were presented at the Maryland Symposium in Elasticity in the spring of 1952. Some hints as to their method may be gleaned, however, by reference to Mindlin [4].

${ }^{*}$ Received March 21, 1953. 
The present paper presents a derivation of some of the consequences of the Timoshenko theory. These same results could be deduced by use of the basic approach of Mindlin and Hermann. However, since the Timoshenko theory has formed the starting point of many investigations (e.g., [5], [6], [7], [8], [9]) an elementary approach based directly and solely on the equations of that theory will be employed here.

The simplicity of the treatment presented here results from the fact that, apart from the last section, it is based directly upon the equations which result from the above when $\alpha^{*}$ alone is eliminated. This is in contrast to the more usual treatments which are based upon the involved displacement equation obtained by eliminating all variables except $y^{*}(x, t)$ from the above set. Some idea of the complexity introduced by this latter procedure can be gathered from an inspection of the displacement equation for uniform beams which is known to be the following:

$$
E I \frac{\partial^{4} y^{*}}{\partial x^{4}}-\left(I_{0}+\frac{E I \rho}{K A G}\right) \frac{\partial^{4} y^{*}}{\partial x^{2} \partial t^{2}}+\frac{I_{0} \rho}{K A G} \frac{\partial^{4} y^{*}}{\partial t^{4}}+\rho \frac{\partial^{2} y^{*}}{\partial t^{2}}=0
$$

The main consequence of the approach in this paper is the fact that the discussion of orthogonality, initial value problems, problems involving external forces and moments, and problems involving time dependent boundary conditions for the Timoshenko theory can be made to parallel step by step the familiar treatment of the classical BernoulliEuler theory. In particular, for the uniform hinged-hinged beam, a complete analytical solution can be given (much of it will be presented here) to all of the above problems. Moreover, for non-uniform beams, the computation of characteristic values by analogue simulation or by other automatic means is much more direct if the system approach is used and the results of this paper can be applied immediately (cf. [9]).

There are of course important points of difference between the Timoshenko theory and that based on equation (1), or upon theories which neglect either the correction due to shear or rotary inertia. The most important of these is the existence in the Timoshenko theory of two sinusoidal modes of different frequencies corresponding to the same spatial factor. These two sets of modes are, moreover, orthogonal to each other in terms of the two term orthogonality relation which forms the kernel of our method. The existence of the second set of modes also makes it possible to satisfy the four initial conditions required by the Timoshenko theory.

Finally, the existence of the second set of modes makes it possible to demonstrate the completeness of the set of particular solutions, sinusoidal in the time, for the above named problems of the Timoshenko theory.

2. Separation of variables and the orthogonality relation. The separation of variables process can be applied directly to (1) but not to either (2-6) or (7). Thus, for example, (7) will separate under the assumption that $y^{*}(x, t)=y(x) T(t)$ if and only if one of the quantities $I_{0}, 1 / K A G$ vanishes. All will, however, admit solutions sinusoidal in time. Thus if we set $y^{*}(x, t)=y(x) e^{i \omega t}, V^{*}(x, t)=V(x) e^{i \omega t}, M^{*}(x, t)=M(x) e^{i \omega t}, \beta^{*}(x, t)=$ $\beta(x) e^{i \omega t}$ and eliminate $\alpha^{*}$ from the system (2-6), it reduces to the following:

$$
\begin{gathered}
\frac{d V}{d x}=\rho \omega^{2} y, \\
\frac{d M}{d x}-V=-I_{0} \omega^{2} \beta,
\end{gathered}
$$




$$
\begin{gathered}
\frac{d \beta}{d x}-\frac{M}{E I}=0, \\
\frac{d y}{d x}-\beta+\frac{V}{K A G}=0 .
\end{gathered}
$$

In order to discuss the orthogonality of solutions sinusoidal in time for a beam of length $L$ we note that integration by parts furnishes the following Lagrange identity appropriate for the system (8-11) in the usual way:

$$
\begin{aligned}
\int_{0}^{L} & \left\{\left[y_{2} \frac{d V_{1}}{d x}-\beta_{2}\left(\frac{d M_{1}}{d x}-V_{1}\right)+M_{2}\left(\frac{d \beta_{1}}{d x}-\frac{M_{1}}{E I}\right)\right.\right. \\
& -V_{2}\left(\frac{d y_{1}}{d x}-\beta_{1}+\frac{V_{1}}{K A G}\right]-\left[y_{1} \frac{d V_{2}}{d x}-\beta_{1}\left(\frac{d M_{2}}{d x}-V_{2}\right)+M_{1}\left(\frac{d \beta_{2}}{d x}-\frac{M_{2}}{E I}\right)\right. \\
& \left.\left.-V_{1}\left(\frac{d y_{2}}{d x}-\beta_{2}+\frac{V_{2}}{K A G}\right)\right]\right\} d x=\left[y_{2} V_{1}-\beta_{2} M_{1}+M_{2} \beta_{1}-V_{2} y_{1}\right]_{0}^{L} .
\end{aligned}
$$

Thus if $y_{i}, V_{i}, \beta_{i}$ and $\omega_{i},(i=1,2)$, represent two solutions of (8-11) with boundary conditions that make the right-hand side of (12) vanish, the following orthogonality relation is obtained, independent of whether the beam is uniform or not:

$$
\left(\omega_{1}^{2}-\omega_{2}^{2}\right) \int_{0}^{L}\left[\rho y_{1} y_{2}+I_{0} \beta_{1} \beta_{2}\right] d x=0 .
$$

Although many boundary conditions are possible, this discussion will be limited to the consideration of free ends where $M^{*}=V^{*}=0$, hinged or simply supported ends where $y^{*}=M^{*}=0$, and built-in ends where $y^{*}=\beta^{*}=0$ (although some authors mistakenly use $y^{*}=\partial y^{*} / \partial x=0$ in the Timoshenko theory) ${ }^{1}$. Thus, from (12) it is apparent that (13) will hold for: (a) the free-free beam; (b) the cantilever beam (i.e., one end built-in, the other free); (c) the hinged-hinged beam; (d) the built-in, built-in beam; and (e) the built-in, hinged beam.

If $I_{0}$ is zero, and $\omega_{1}^{2} \neq \omega_{2}^{2}$, the relation (13) reduces to

$$
\int_{0}^{L} \rho y_{1} y_{2} d x=0
$$

which is identical to that of the usual textbook theory. This shows that the displacements associated with different modes are orthogonal with respect to the weighting function $\rho(x)$.

Even if $I_{0}$ is not zero, the relation (13) is readily interpreted. As we shall see in $\$ 6$ the solution of the initial and boundary value problem will, by our method, reduce to the determination of a set of constants $C_{n}(n=1,2, \cdots)$ such that two given functions $f(x)$ and $g(x)$ admit the simultaneous expansion

$$
f(x)=\sum_{n=1}^{\infty} C_{n} y_{n}(x), \quad g(x)=\sum_{n=1}^{\infty} C_{n} \beta_{n}(x) .
$$

\footnotetext{
${ }^{1}$ For a discussion of this point as well as of the orthogonality relation for the cases where either the shear or rotary inertia term is neglected, see [10].
} 
In order to show that this is possible in virtue of the relation (13), we introduce two mutually perpendicular unit vectors $\mathbf{e}_{1}$ and $\mathbf{e}_{2}$ and consider formal vectors of the form

$$
\mathbf{A}(x)=a_{1}(x) \mathbf{e}_{1}+a_{2}(x) \mathbf{e}_{2},
$$

with a scalar product defined by

$$
\int_{0}^{L} \mathbf{A}(x) \cdot \mathbf{B}(x) d x=\int_{0}^{L}\left[\rho a_{1}(x) b_{1}(x)+I_{0} a_{2}(x) b_{2}(x)\right] d x .
$$

If in particular we let

$$
\mathbf{K}(x)=f(x) \mathbf{e}_{1}+g(x) \mathbf{e}_{2}, \quad \mathbf{Z}_{p}(x)=y_{p}(x) \mathbf{e}_{1}+\beta_{p}(x) \mathbf{e}_{2},
$$

then we must determine the constants $\left\{C_{p}\right\}$ in the vector expansion

$$
\mathbf{K}(x)=\sum_{p=1}^{\infty} C_{p} \mathbf{Z}_{p}(x) .
$$

Taking the scalar product in the sense defined by (15) we find that

$$
C_{p}=\frac{\int_{0}^{L}\left[\rho f(x) y_{p}(x)+I_{0} g(x) \beta_{p}(x)\right] d x}{\int_{0}^{L}\left[\rho\left\{y_{p}(x)\right\}^{2}+I_{0}\left\{\beta_{p}(x)\right\}^{2}\right] d x}, \quad(p=1,2, \cdots) .
$$

The situation here is somewhat special in that for a general fourth order system one would expect that it would be necessary to introduce four mutually perpendicular unit vectors and a scalar product of the form

$$
\int_{0}^{L} \mathbf{Z}_{1}(x) \cdot \mathbf{Z}_{2}(x) d x=\int_{0}^{L}\left[\rho y_{1} y_{2}+I_{0} \beta_{1} \beta_{2}+A M_{1} M_{2}+B V_{1} V_{2}\right] d x
$$

Because the last two terms are absent from (13) one cannot in any real sense say that the vector solutions $y_{k}, V_{k}, M_{k}$ and $\beta_{k}$, corresponding to $\omega_{k}^{2}$, are orthogonal to each other for different $k$ 's but only that the first two components of different solutions have this property. From this point of view the relation which obtains if $I_{0}$ vanishes is even more special in that then only the first components are orthogonal. ${ }^{2}$

3. Normal modes of the uniform beam. If the beam is uniform the system (8-11) can be treated by standard methods. Thus if $y(x)=y_{0} e^{\lambda x}, M(x)=M_{0} e^{\lambda x}, V(x)=$ $V_{0} e^{\lambda x}, \beta(x)=\beta_{0} e^{\lambda x}$ where $y_{0}, V_{0}, M_{0}$ and $\beta_{0}$ are constants, and if the notation $a=1 / E I$ and $b=1 / K A G$ is used the system (8-11) reduces, after division by $e^{\lambda x}$, to

$$
\begin{array}{ll}
\lambda V_{0}-\rho \omega^{2} y_{0}=0, & \lambda M_{0}-V_{0}+I_{0} \omega^{2} \beta_{0}=0, \\
\lambda \beta_{0}-a M_{0}=0, & \lambda y_{0}-\beta_{0}+b V_{0}=0 .
\end{array}
$$

This system can have a non-trivial solution if and only if the determinant of the coefficients vanishes; explicitly, if

$$
\lambda^{4}+\left(a I_{0}+b \rho\right) \lambda^{2} \omega^{2}-a \rho \omega^{2}+a b \rho I_{0} \omega^{4}=0,
$$

from which it follows that the roots $\lambda$ satisfy the equation

$$
2 \lambda^{2}=-\left(a I_{0}+b \rho\right) \omega^{2} \pm\left\{\left(a I_{0}+b \rho\right)^{2} \omega^{4}+4 a \rho \omega^{2}-4 a b \rho I_{0} \omega^{4}\right\}^{1 / 2} .
$$

${ }^{2} M, V$ may however be eliminated as in $\$ 8$. 
There are several possible cases associated with this characteristic equation. If $\omega=0$ then all of the roots are zero and the system (8-11) simplifies in that its right-hand members vanish. It is readily verified that the solution in this special case is the following:

$$
\begin{gathered}
y=C_{1}^{0}+C_{2}^{0} x+C_{3}^{0} x^{2}+C_{4}^{0} x^{3}, \quad V=\frac{6 C_{4}^{0}}{a}, \\
M=\frac{2 C_{3}^{0}+6 C_{4}^{0} x}{a}, \quad=\left(C_{2}^{0}-\frac{6 b}{a} C_{4}^{0}\right)+2 C_{3}^{0} x+3 C_{4}^{0} x^{2} .
\end{gathered}
$$

If $\omega \neq 0$ but $\omega^{2}=1 / b I_{0}$ then $\lambda=0$ is a double root and the other two roots are purely imaginary and conjugates of each other. This case cannot be admitted into the theory of the system (8-11) since it implies that the relations for $V$ and $\beta$ in terms of $y$ that can be deduced from (8-11) are meaningless (cf. [9]).

For reasonable physical beams, the condition $\omega^{2}<1 / b I_{0}$ is valid for the frequencies of interest. Under this assumption we obtain the four roots:

$$
\lambda_{1}, \lambda_{2}= \pm \gamma, \quad \lambda_{3}, \lambda_{4}= \pm i \epsilon,
$$

where

$$
2^{1 / 2}\{\underline{\gamma}\}=\left\{-\left(a I_{0}+b \rho\right) \omega^{2} \pm\left[\left(a I_{0}-b \rho\right)^{2} \omega^{4}+4 a \rho \omega^{2}\right]^{1 / 2}\right\}^{1 / 2}
$$

From this it is readily deduced that the most general solution of the system (8-11) must be, for fixed $\omega$, of the form

$$
\begin{aligned}
& y=C_{1} \cosh \gamma x+C_{2} \sinh \gamma x+C_{3} \cos \epsilon x+C_{4} \sin \epsilon x, \\
& V=\frac{\rho \omega^{2}}{\gamma}\left\{C_{1} \sinh \gamma x+C_{2} \cosh \gamma x\right\}+\frac{\rho \omega^{2}}{\epsilon}\left\{C_{3} \sin \epsilon x-C_{4} \cos \epsilon x\right\}, \\
& M=\frac{b \rho \omega^{2}+\gamma^{2}}{a}\left\{C_{1} \cosh \gamma x+C_{2} \sinh \gamma x\right\}+\frac{b \rho \omega^{2}-\epsilon^{2}}{a}\left\{C_{3} \cos \epsilon x+C_{4} \sin \epsilon x\right\}, \\
& \beta=\frac{b \rho \omega^{2}+\gamma^{2}}{\gamma}\left\{C_{1} \sinh \gamma x+C_{2} \cosh \gamma x\right\}+\frac{b \rho \omega^{2}-\epsilon^{2}}{\epsilon}\left\{C_{3} \sin \epsilon x-C_{4} \cos \epsilon x\right\} .
\end{aligned}
$$

4. Normal modes of the uniform hinged-hinged beam. In order to find the modes of oscillation in the cases of physical interest, boundary conditions need to be imposed on the equations (19). By far the simplest case and the only one which seems capable of complete analytical treatment without approximations is that of the hinged-hinged beam where $y^{*}=M^{*}=0$ holds at $x=0$ and $x=L$. Imposing this boundary condition at $x=0$ we obtain the equations

$$
C_{1}+C_{3}=0, \quad \frac{b \rho \omega^{2}+\gamma^{2}}{a} C_{1}+\frac{b \rho \omega^{2}-\epsilon^{2}}{a} C_{3}=0 .
$$

Since the determinant of this system has the non-zero value $-\left(\gamma^{2}+\epsilon^{2}\right) / a$, it follows that $C_{1}=C_{3}=0$. It is convenient at this point to simplify the formulas by selecting the unit of length so that the beam is of length $\pi$. The formulas for a beam of arbitrary length can be obtained by replacing $\gamma \pi$ and $\epsilon \pi$ by $\gamma L$ and $\epsilon L$ respectively in the relations 
$(20,21)$ and $n$ by $n \pi / L$ in all succeeding formulas. The boundary conditions at $x=\pi$ yield the equations

$$
\begin{gathered}
C_{2} \sinh \gamma \pi+C_{4} \sin \epsilon \pi=0, \\
\left(b \rho \omega^{2}+\gamma^{2}\right) C_{2} \sinh \gamma \pi+\left(b \rho \omega^{2}-\epsilon^{2}\right) C_{4} \sin \epsilon \pi=0 .
\end{gathered}
$$

In order to have a non-trivial solution the determinant of this system must vanish. Thus one obtains the equation

$$
\left(\gamma^{2}+\epsilon^{2}\right) \sinh \gamma \pi \sin \epsilon \pi=0 .
$$

Since $\gamma$ and $\epsilon$ as defined by (18) are real, the only roots of interest are simply those for which

$$
\gamma \neq 0, \quad \epsilon=n, \quad(n=0,1,2, \cdots),
$$

for if $\gamma$ and $\epsilon$ were both zero, then $\omega$ must be zero and the special solution of the system (8-11) must be employed. It is readily verified that there exist no non-zero constants $C_{k}^{0}(k=1,2,3,4)$, which permit the boundary conditions to be satisfied for $\omega=0$. On the other hand, the combination $\gamma=0$ and $\epsilon=n$ cannot occur, since this would imply that $b I_{0} \omega^{2}=1$.

The corresponding spatial parts are therefore of the form

$$
\begin{aligned}
y & =C_{n} \sin n x, & M & =C_{n}\left(\frac{b \rho \omega^{2}-n^{2}}{a}\right) \sin n x, \\
V & =C_{n}\left(-\frac{\rho \omega^{2}}{n}\right) \cos n x, & \beta & =C_{n}\left(\frac{n^{2}-b \rho \omega^{2}}{n}\right) \cos n x,
\end{aligned}
$$

for $n=1,2, \cdots$.

It should be noted that for the uniform hinged-hinged beam the assumption that $b I_{0} \omega^{2}<1$ is really unnecessary, for if $b I_{0} \omega^{2}>1$ it follows that all four roots of (17) would be imaginary so that the secular determinant would involve the product $\sin \gamma \pi \sin \epsilon \pi$. This product does not introduce any new spatial eigenfunctions.

The values of $\omega$ which correspond to $\epsilon=n$ are obtained by setting $\lambda=i \epsilon=i n$ in equation (17) and solving the resulting equation for $\omega$. Thus, from

we obtain

$$
\omega^{4}-\left[\frac{a \rho+\left(a I_{0}+b \rho\right) n^{2}}{a b \rho I_{0}}\right] \omega^{2}+\frac{n^{4}}{a b \rho I_{0}}=0
$$

$$
\omega^{2}=\frac{\left[a \rho+\left(a I_{0}+b \rho\right) n^{2}\right] \pm\left\{\left[a \rho+\left(a I_{0}+b \rho\right) n^{2}\right]^{2}-4 a b \rho I_{0} n^{4}\right\}^{1 / 2}}{2 a b \rho I_{0}}
$$

so that corresponding to the spatial functions (22) there are two distinct values of $\omega^{2}$. Let the roots be denoted by $\pm \omega_{1 n}$ and $\pm \omega_{2 n}$. We shall assume $\left|\omega_{1 n}\right|<\left|\omega_{2 n}\right|$. For fixed $n$, we therefore obtain particular solutions to the system (8-11) of the form

$$
\begin{aligned}
y= & \sin n x\left\{E_{i n} \cos \omega_{1 n} t+F_{i n} \sin \omega_{i n} t\right\}, \\
V= & -\cos n x\left[-\rho \omega_{i n}^{2} / \mathrm{n}\right]\left[E_{i n} \cos \omega_{i n} t+F_{i n} \sin \omega_{i n} t\right], \\
M= & \sin n x\left[\left(b \rho \omega_{i n}^{2}-n^{2}\right) / a\right]\left[E_{i n} \cos \omega_{i n} t+F_{i n} \sin \omega_{i n} t\right], \\
\beta= & -\cos n x\left[\left(b \rho \omega_{i n}^{2}-n^{2}\right) / n\right]\left[E_{i n} \cos \omega_{i n} t+F_{i n} \sin \omega_{i n} t\right] ; \\
& (i=1,2 ; n=1,2, \cdots) .
\end{aligned}
$$


Consideration of the second mode which occurs for $n$ fixed seems to have been entirely overlooked by Timoshenko [2] in his original paper, although he treated only the uniform hinged-hinged beam.

For different values of $n$ it is clear that orthogonality results, since the general relation (13) is satisfied trivially inasmuch as the integrals

$$
\rho \int_{0}^{\pi} y_{1} y_{2} d x, \quad I_{0} \int_{0}^{\pi} \beta_{1} \beta_{2} d x
$$

vanish separately. We will now investigate how the relation (13) is satisfied for the same $n$ but for the two modes which correspond to $\omega_{1 n}$ and $\omega_{2 n}$. In this case, Equation (13) is explicitly

$$
\begin{aligned}
\int_{0}^{\pi}\left(\rho y_{1} y_{2}+I \beta_{1} \beta_{2}\right) d x=K \int_{0}^{\pi}\{ & \rho \sin ^{2} n x \\
& \left.+\frac{I_{0}}{n^{2}}\left[b^{2} \rho^{2} \omega_{1 n}^{2} \omega_{2 n}^{2}-b \rho n^{2}\left(\omega_{1 n}^{2}+\omega_{2 n}^{2}\right)+n^{4}\right] \cos ^{2} n x\right\} d x,
\end{aligned}
$$

where $K$ is some arbitrary constant.

The coefficient of $\cos ^{2} n x$ in this expression can be simplified by noting that it follows from (23) that

and that

$$
\omega_{1 n}^{2}+\omega_{2 n}^{2}=\frac{a \rho+\left(a I_{0}+b \rho\right) n^{2}}{a b \rho I_{0}}
$$

$$
\omega_{1 n}^{2} \omega_{1 n}^{2}=\frac{n^{4}}{a b \rho I_{0}}
$$

Thus

$$
\frac{I_{0}}{n^{2}}\left[b^{2} \rho^{2} \omega_{1 n}^{2} \omega_{2 n}^{2}-b \rho n^{2}\left(\omega_{1 n}^{2}+\omega_{2 n}^{2}\right)+n^{4}\right]=-\rho,
$$

so that

$$
\int_{0}^{\pi}\left(\rho y_{1} y_{2}+I_{0} \beta_{1} \beta_{2}\right) d x=K \rho \int_{0}^{\pi}\left(\sin ^{2} n x-\cos ^{2} n x\right) d x=-K \rho \int_{0}^{\pi} \cos 2 n x d x=0 .
$$

Thus the modes are orthogonal in accordance with (13) if they correspond either to different $n$ 's and the same $\omega$ or to the same $n$ and to different $\omega$ 's. It is necessary to use both sets of modes for a fixed $n$ in order to obtain a solution to the general initial and boundary value problem.

5. Comparison of universal curves for hinged-hinged and free-free beams. In order that the shear and rotary inertia frequency effects may be estimated for any uniform hinged-hinged beam, it is convenient to introduce dimensionless parameters. Such a set of parameters has already been introduced by Howe et al. [9] and an electronic differential analyzer has been used to compute a set of curves showing the effects of rotary inertia and shear on a uniform free-free beam. Since these authors have kindly granted permission to reproduce their curves, it seemed expedient to use the same parameters and to compute the corresponding values for the uniform hinged-hinged beam for which the exact theory presented here is available. 
The dimensionless parameters introduced by Howe et al. were defined as follows:

$$
S=E I / K A G L^{2}, \quad N=K G / E, \quad \lambda=\omega L^{2}(\rho / E I)^{1 / 2} .
$$

If the effects of shear forces and rotary inertia are both negligible, then $S=N=0$. This will be the case if the length of the beam is very long compared with its thickness; such an idealized beam is often termed "infinitely long". If $\lambda_{0}$ is used to denote the value of the appropriate mode of the "infinitely long" beam, the ratio $\lambda / \lambda_{0}$ is a convenient measure of the change in frequency caused by values of $S$ and $N$ different from zero.

For the uniform hinged-hinged beam, $\lambda_{h}$ may be computed directly from equation (23) which may be written in the form

$$
\lambda_{h}^{4} S^{2} N-\left[1+S(1+N) m^{2} \pi^{2}\right] \lambda_{h}^{2}+m^{4} \pi^{4}=0, \quad(m=1,2, \cdots),
$$

so that

$$
\begin{aligned}
\lambda_{h^{*}}=(2 N)^{-1 / 2}\left[S^{-2}\right. & +S^{-1}(1+N) m^{2} \pi^{2} \\
& \left. \pm\left\{\left[S^{-2}+S^{-1}(1+N) m^{2} \pi^{2}\right]^{2}-4 S^{-2} m^{4} \pi^{4} N\right\}^{1 / 2}\right]^{1 / 2} .
\end{aligned}
$$

The corresponding values of $\lambda_{0}$ are computed from (25) by setting $S=N=0$ so that $\lambda_{0}=m^{2} \pi^{2}$.

The smallest values of $100 \lambda_{h} / \lambda_{0}$ for a particular mode are plotted versus 1 'S in Figure 1 for values of $N=0,0.1,0.2$, and 0.3 . The corresponding values for a uniform

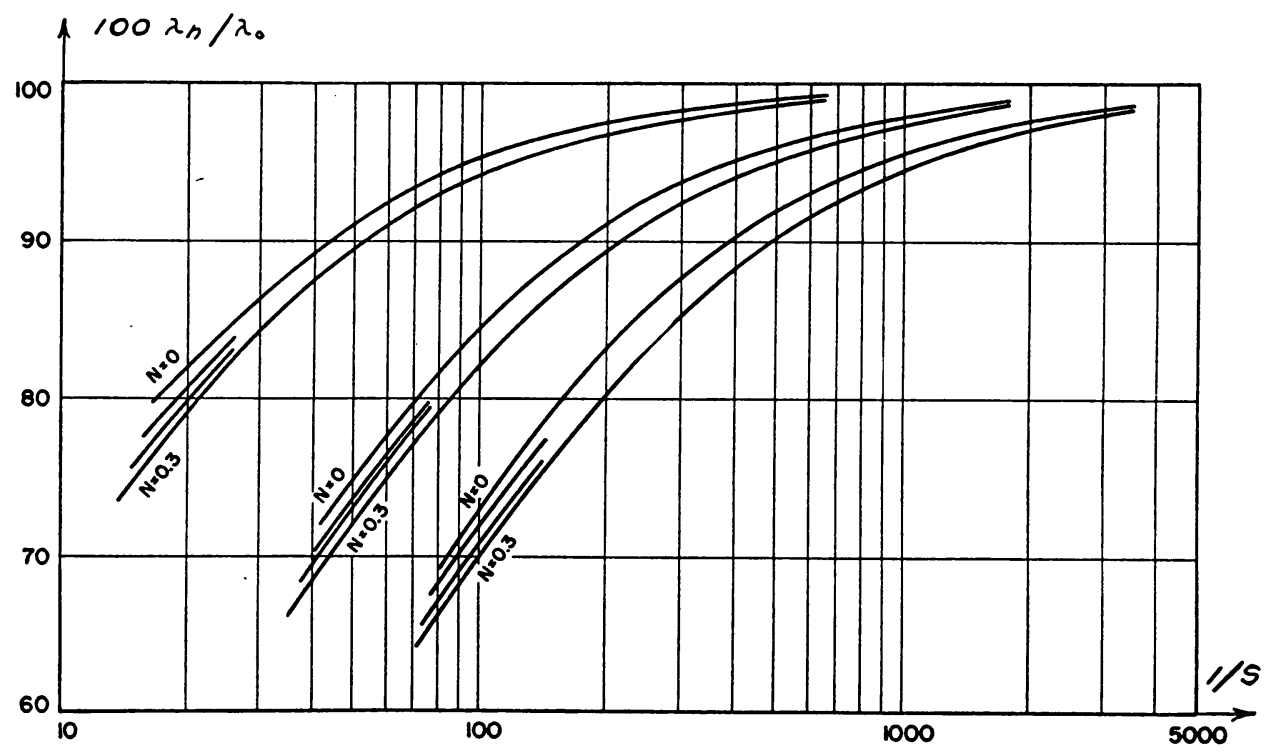

FIg. 1

free-free beam as given in Howe et al. [9] appear in Figure 2. Figure 3 is a similar graph for the values of $\lambda_{h} / \lambda_{0}$ as determined by the plus sign in equation (26) for the uniform hinged-hinged beam.

These curves may be used to estimate either separately or simultaneously the effects of shear and rotary inertia upon the frequency of vibration. It is apparent from its definition that $S$ increases with an increase in flexural rigidity, and decreases with an 


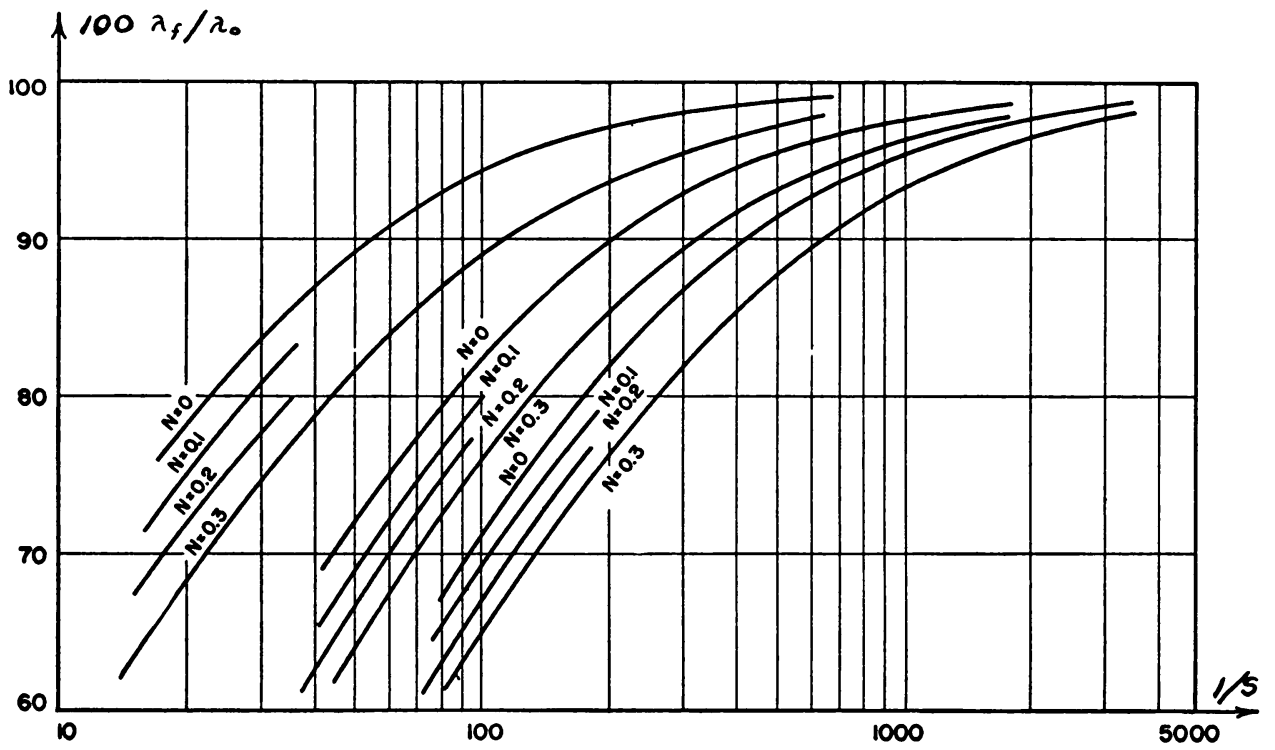

Fig. 2

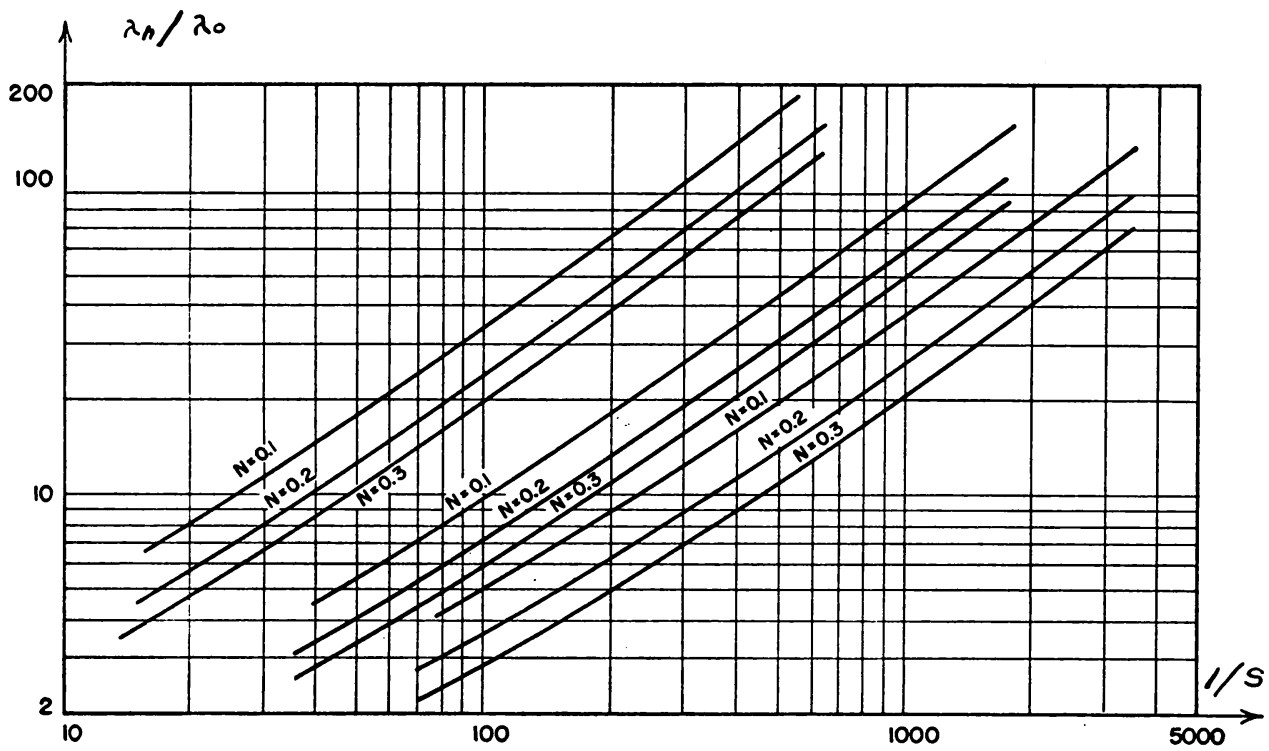

FIG. 3

increase in shear rigidity or an increase in length. It is therefore a parameter capable of describing the relative importance of shear rigidity for beams of given flexural rigidity and of given length. On the other hand, the expression for $N$ may be written as

$$
N=K A G I_{0} / E I \rho
$$

and is therefore seen to increase with an increase in shear rigidity or rotary inertia and to decrease with an increase in flexural rigidity. Thus, $N$ is a suitable parameter to measure the relative effect of rotary inertia for beams of given shear and flexural rigidity. Alter- 
nately, since $I_{0}$ is directly proportional to the density, it may be used to measure the relative importance of the size of the cross-section.

6. The initial value problem for the hinged-hinged beam. An examination of the system (2-6) shows that the general initial value problem requires the specification of $y^{*}(x, 0), y_{t}^{*}(x, 0), \beta^{*}(x, 0)$, and $\beta_{t}^{*}(x, 0)$ in addition to the boundary conditions appropriate to the type of beam under consideration. Let us write

$$
\begin{array}{ll}
y^{*}(x, 0)=f_{1}(x), & \beta^{*}(x, 0)=g_{1}(x), \\
y_{t}^{*}(x, 0)=f_{2}(x), & \beta_{t}^{*}(x, 0)=g_{2}(x) .
\end{array}
$$

Then, because of the linearity of the system (2-6), a superposition of the solution (24) will satisfy this sytem and the boundary conditions for the hinged-hinged beam. Such a sum is of the following form:

$$
\begin{aligned}
& y^{*}(x, t)=\sum_{i=1}^{2} \sum_{n=1}^{\infty} \sin n x\left[E_{i n} \cos \omega_{i n} t+F_{i n} \sin \omega_{i n} t\right], \\
& \beta^{*}(x, t)=\sum_{i=1}^{2} \sum_{n=1}^{\infty} \cos n x\left(b \rho \omega_{i n}^{2}-n^{2}\right) n^{-1}\left[E_{i n} \cos \omega_{i n} t+F_{i n} \sin \omega_{i n} t\right],
\end{aligned}
$$

where $M^{*}(x, t), V^{*}(x, t)$ can be expressed in a similar fashion. In order to satisfy the initial conditions (28), the coefficients $E_{i n}, F_{i n},(i=1,2 ; n=1,2, \cdots)$, must be determined from the following series representation:

$$
\begin{aligned}
& f_{1}(x)=\sum_{n=1}^{\infty} E_{1 n} \sin n x+\sum_{n=1}^{\infty} E_{2 n} \sin n x, \\
& g_{1}(x)=-\sum_{n=1}^{\infty} E_{1 n}\left(b \rho \omega_{1 n}^{2}-n^{2}\right) n^{-1} \cos n x-\sum_{n=1}^{\infty} E_{2 n}\left(b \rho \omega_{2 n}^{2}-n^{2}\right) n^{-1} \cos n x, \\
& f_{2}(x)=\sum_{n=1}^{\infty}\left(\omega_{1 n} F_{1 n}\right) \sin n x+\sum_{n=1}^{\infty}\left(\omega_{2 n} F_{2 n}\right) \sin n x, \\
& g_{2}(x)=-\sum_{n=1}^{\infty}\left(\omega_{1 n} F_{1 n}\right)\left(b \rho \omega_{1 n}^{2}-n^{2}\right) n^{-1} \cos n x-\sum_{n=1}^{\infty}\left(\omega_{2 n} F_{2 n}\right)\left(b \rho \omega_{2 n}^{2}-n^{2}\right) n^{-1} \cos n x .
\end{aligned}
$$

Although the coefficients $E_{1 n}, E_{2 n}, F_{1 n}$, and $F_{2 n}$ can be evaluated in this case directly by the usual Fourier methods, the orthogonality relation (13) and its interpretation offers the advantage of furnishing these values directly without the necessity of solving two sets of algebraic equations. Thus, for example,

$$
E_{1 n}=\frac{2}{\pi} \frac{\int_{0}^{\pi}\left[f_{1}(x) \sin n x-I_{0} n^{-1} \rho^{-1}\left(b \rho \omega_{1 n}^{2}-n^{2}\right) g_{1}(x) \cos n x\right] d x}{1+\rho^{-1} n^{-2} I_{0}\left(b \rho \omega_{1 n}^{2}-n^{2}\right)^{2}} .
$$

If the usual Fourier coefficients are employed one finds

$$
E_{1 n}=\frac{2}{\pi} \frac{\int_{0}^{\pi}\left[f_{1}(x) \sin n x+n\left(b \rho \omega_{2 n}^{2}-n^{2}\right)^{-1} g_{1}(x) \cos n x\right] d x}{b \rho\left(\omega_{2 n}^{2}-\omega_{1 n}^{2}\right)\left[b \rho\left(\omega_{2 n}^{2}-n^{2}\right)\right]^{-1}} .
$$

The equality of this with the first expression for $E_{1 n}$ follow directly since

$$
n /\left(b \rho \omega_{2 n}^{2}-n^{2}\right)=-I_{0} b \rho\left(\omega_{1 n}^{2}-n^{2}\right) / n \rho,
$$


and

$$
\begin{aligned}
\left(b \rho \omega_{2 n}^{2}-\omega_{1 n}^{2}\right) /\left(b \rho \omega_{2 n}^{2}-n^{2}\right) & =1+\left(n^{2}-b \rho \omega_{1 n}^{2}\right) /\left(b \rho \omega_{2 n}^{2}-n^{2}\right) \\
& =1+I_{0}\left(b \rho \omega_{1 n}^{2}-n^{2}\right)^{2} / \rho n^{2},
\end{aligned}
$$

in virtue of the sum and product of the roots $\omega_{1 n}^{2}$ and $\omega_{2 n}^{2}$ of (23).

The rigorous justification of the above expansions as solutions of the initial value problem would, in general, involve a proof of the completeness of the set of eigenfunctions and a study of the convergence properties of these expansions. Here, because of the identity of these expansions with those of usual Fourier sine and cosine series, it is well known that the set of functions is complete and many conditions sufficient to guarantee the convergence of such series are, of course, known. Moreover, since the general solution of the system (8-11) for $\omega=0$ does not admit a special solution satisfying the boundary conditions and allowing $V^{*}$ and $\beta^{*}$ to be constant, the identity of these expansions with those of Fourier series can be used to deduce the fact that the functions $g_{1}(x)$ and $g_{2}(x)$ cannot be specified arbitrarily but must satisfy the additional conditions that

$$
\int_{0}^{\pi} g_{i}(x) d x=0 \quad \text { for } i=1,2 .
$$

This conclusion is however a function of the boundary conditions. For example, for a free-free beam, the solution $\beta=$ constant is permitted by the system (8-11) if $\omega=0$. For the uniform hinged-hinged beam it is clear that these constraints on $g_{i}(x)$ are merely symmetry requirements since $\beta$ is the slope due to bending.

7. Beam problems involving external forces and moments. If an external force $F^{*}(x, t)$ per unit length and an external moment $G^{*}(x, t)$ per unit length is allowed to act on a non-uniform beam, only the first two differential equations of the system (2-6) are modified and they become

$$
\rho \frac{\partial^{2} y^{*}}{\partial t^{2}}+\frac{\partial V^{*}}{\partial x}=F^{*}(x, t), \quad-\frac{\partial M^{*}}{\partial x}+I_{0} \frac{\partial^{2} \beta^{*}}{\partial t^{2}}+V^{*}=G^{*}(x, t) .
$$

The solution of any problem of this type may be obtained by the superposition of solutions of the corresponding problem in which the external forces and moments are zero. It is merely necessary to seek solutions of the form

$$
\begin{aligned}
y^{*}=\int_{0}^{t} y^{\prime}(x, t-\sigma) d \sigma, & V^{*}=\int_{0}^{t} V^{\prime}(x, t-\sigma) d \sigma, \\
M^{*}=\int_{0}^{t} M^{\prime}(x, t-\sigma) d \sigma, & \beta^{*}=\int_{0}^{t} \beta^{\prime}(x, t-\sigma) d \sigma,
\end{aligned}
$$

and to impose the following initial conditions on the functions $y^{\prime}(x, \tau)$ and $\beta^{\prime}(x, \tau)$ where $\tau=t-\sigma$ :

$$
\begin{aligned}
& y^{\prime}(x, 0)=0, \\
& y^{\prime}(x, 0)=\left.y_{t}^{\prime}(x, t-\sigma)\right|_{\sigma=t}=F^{*}(x, \sigma), \\
& \beta^{\prime}(x, 0)=0, \quad \beta_{t}^{\prime}(x, 0)=\left.\beta^{\prime}(x, t-\sigma)\right|_{\sigma-t}=G^{*}(x, \sigma) .
\end{aligned}
$$


It now follows that the functions $y^{\prime}, M^{\prime}, V^{\prime}$ and $\beta^{\prime}$ satisfy the free system (2-6) and that if they satisfy a given set of boundary conditions then the function $y^{*}, M^{*}, V^{*}$ and $\beta^{*}$ defined by (29) will satisfy the same boundary conditions. In order to determine the functions $y^{\prime}, M^{\prime}, V^{\prime}$ and $\beta^{\prime}$ it is merely necessary to solve a special case of the initial value problem corresponding to the given set of boundary conditions and the initial conditions (30) as a function of the parameter.

8. Beam vibrations with time dependent boundary conditions. In order to reduce a beam problem for the system (2-6) which involves time dependent boundary conditions to manageable form, it seems simplest to eliminate $M^{*}$ and $V^{*}$ from the system (2-6) to obtain the equations

$$
\begin{aligned}
& L_{1}\left(y^{*}, \beta^{*}\right)=\rho \frac{\partial^{2} y^{*}}{\partial t^{2}}+\frac{\partial}{\partial x}\left[K A G\left(\beta^{*}-\frac{\partial y^{*}}{\partial x}\right)\right]=0, \\
& L_{2}\left(y^{*}, \beta^{*}\right)=-\frac{\partial}{\partial x}\left(E I \frac{\partial \beta^{*}}{\partial x}\right)+I_{0} \frac{\partial^{2} \beta^{*}}{\partial t^{2}}+K A G\left(\beta^{*}-\frac{\partial y^{*}}{\partial x}\right)=0
\end{aligned}
$$

and the following expressions for $M^{*}$ and $V^{*}$ :

$$
M^{*}=E I \frac{\partial \beta^{*}}{\partial x}, \quad V^{*}=K A G\left(\beta^{*}-\frac{\partial y^{*}}{\partial x}\right) .
$$

Now if $y^{*}$ and $\beta^{*}$ are decomposed to the relations

$$
y^{*}=y_{0}^{*}+y_{1}^{*}, \quad \beta^{*}=\beta_{0}^{*}+\beta_{1}^{*}
$$

these become

$$
\begin{array}{cc}
L_{1}\left(y_{0}^{*}, \beta_{0}^{*}\right)=-L_{1}\left(y_{0}^{*}, \beta_{1}^{*}\right), & L_{2}\left(y_{0}^{*}, \beta_{0}^{*}\right)=-L_{2}\left(y_{1}^{*}, \beta_{1}^{*}\right), \\
M^{*}=M_{0}^{*}+M_{1}^{*}, & V^{*}=V_{0}^{*}+V_{1}^{*} .
\end{array}
$$

If, following Mindlin and Goodman [11], one sets

$$
y_{1}^{*}=f_{1}(t) h_{1}(x)+f_{2}(t) h_{2}(x), \quad \beta_{1}^{*}=g_{1}(t) k_{1}(x)+g_{2}(t) k_{2}(x),
$$

where the functions $f_{i}(x), g_{i}(x), h_{i}(t)$ and $k_{i}(t)$ are arbitrary for $i=1,2$, it is always possible to choose these eight functions in such a way as to reduce the boundary value problem for $y_{0}^{*}, \beta_{0}^{*}, V_{0}^{*}$, and $M_{0}^{*}$ to one involving time independent boundary conditions, known initial conditions, and known external forces and moments as given by the right-hand sides of $(31,32)$. This problem can in turn be decomposed into two problems of the types created in $\$ 6$ and $\$ 7$ respectively and thus a general solution can be obtained by superposition.

Acknowledgements. The author would like to thank his colleagues, Professors R. V. Churchill, G. E. Hay, and J. Ormondroyd as well as Professor R. D. Mindlin of Columbia University for many helpful suggestions and criticisms.

\section{BIBLIOGRAPHY}

1. Lord Rayleigh, The theory of sound, MacMillan Co., London, 1877, par. 186.

2. S. P. Timoshenko, On the correction for shear of the differential equation for transterse vibration of prismatic bars, London Phil. Mag. (6) 41, 744 (1921).

3. 43, 125 (1921). 
4. R. D. Mindlin, Influence of rotary inertia and shear on flectural motions of isotropic, elastic plates, J. Appl. Mech. 18, 31 (1951).

5. E. Goens, $\ddot{C} b e r$ die Bestimmung der Elastizitätsmoduls von Stäben mit Hilfe von Biegungsschwingungen, Ann. Physik. (5) 11, 649 (1931).

6. J. Ormondroyd, R. Hess and G. Hess, Theoretical research on the dynamics of a ship's structure, Univ. of Mich. Eng. Res. Inst., Third Progress Report, Office of Naval Research Contract n50-ri-116 (1949).

7. R. Hess, Theoretical vibrations of beams, Ph. D. thesis, Dept. of Eng. Mechanics, Univ. of Mich., 1949.

8. E. T. Kruszewski, Effect of transverse shear and rotary inertia on the natural frequency of a uniform beam, NACA, Tech. Note 1909 (1949).

9. C. Howe, R. Howe, and L. Rauch, Application of the electronic differential analyzer to the oscillation of beams, including shear and rotary inertia, Ext. Memo. UMM-67, Univ. of Mich. Res. Inst., (1951).

10. C. L. Dolph, Normal modes of oscillation of beams, Ext. Memo. UMM-79, Univ. of Mich. Res. Inst. (1950).

11. R. Mindlin and L. E. Goodman, Beam vibrations with time dependent boundary conditions, J. App. \ech. 17, 377 (1950). 